\title{
Structure of Normal and Contracted Tail Sheaths of T4 Bacteriophage
}

\author{
S. KRIMM \\ Department of Physics, and Biophysics Research Division \\ Institute of Science and Technology, University of Michigan \\ Ann Arbor, Michigan, U.S.A. \\ AND \\ T. F. ANDerson \\ Institute for Cancer Research \\ Philadelphia, Pennsylvania, U.S.A.
}

(Received 4 October 1966, and in revised form 11 February 1967)

\begin{abstract}
The structural arrangement of protein subunits in extended and contracted tail sheaths of $\mathrm{T} 4$ bacteriophage has been studied by optical diffractometry of electron micrographs. The analysis of such diffraction patterns shows that the extended sheath consists of annuli of six subunits, these being arranged in a helix of close to seven annuli in two turns. The annulus repeat in the helix direction is $38 \cdot 1 \AA$. For the contracted sheath the axial periodicity is found to be $32 \AA$, which must arise from twice the repeat of the extended sheath. The symmetry of the contracted sheath diffraction pattern is consistent with a helix of five annuli (of 12 subunits) in one turn. Such a structure is seen to arise simply from the extended sheath by the merging of pairs of annuli. The dimensional changes suggested by the above structures indicate that contraction is accompanied by a conformational change in the subunit. The pairing of annuli may have to be a progressive one in order not to isolate single annuli, thus being an example of what could be called a "domino reaction".
\end{abstract}

\section{Introduction}

The application of the negative-staining technique (Brenner \& Horne, 1959) to the electron-microscopic study of bacteriophages has revealed much about the fine structure of these viruses. In particular, detailed structure in the tail sheath of the T-even phages was first demonstrated by this method (Brenner et al., 1959). Subsequent olectron microscope studies (Anderson, 1960; Daems, Van de Pol \& Cohen, 1961; Fernández-Morán, 1962; Bradley, 1963; Kellenberger \& Boy de la Tour, 1964) have confirmed the original structural conclusions of Brenner et al. (1959). These indicate that the extended sheath is roughly $850 \AA$ long and 160 to $170 \AA$ in outer diameter, and contains 24 or 25 striations of spacing 35 to $40 \AA$. Upon contraction, the tail sheath becomes about $350 \AA$ long, 260 to $270 \AA$ in outer diameter, and exhibits roughly 12 striations along its length. The striations in the extended sheath are assumed to be annuli with an inner diameter of about $70 \AA$. These annuli exhibit a substructure, and it has been proposed that the subunits in each annulus are related to the particles of molecular weight about 54,000 (Brenner et al., 1959) 
or 55,500 (Sarkar, Sarkar \& Kozloff, 1964) which can be obtained from the tail sheath. The number of subunits per annulus cannot be measured easily, but it has been inferred (Bradley, 1963; Kellenberger \& Boy de la Tour, 1964) to be six, a number compatible with the known hexagonal symmetry of the base-plate which is attached to the terminal annulus (Anderson \& Stephens, 1964). Nor can the helical symmetry relating one annulus to the next be readily determined from the electron micrographs, although such symmetry has been suggested and possible models of the tail sheath structure proposed (Bradley, 1963; Kellenberger \& Boy de la Tour, 1964).

In order to elucidate these points and to determine the nature of the structural change on contraction, we have undertaken an optical diffraction analysis of electron micrographs of tail sheaths. The optical diffraction technique is uniquely suited to revealing all of the harmonic components of a structure (Taylor \& Lipson, 1964), and it has been successfully applied to such study of electron micrographs of viruses and their components (Klug \& Berger, 1964).

\section{Results and Discussion}

Electron micrographs were obtained of bacteriophage T4, both with extended and contracted tails. The specimens were negatively stained with sodium silicotungstate at $\mathbf{p H} 7$.

Optical diffraction patterns were obtained of the tail sheaths, after appropriate masking of the original micrograph or photographic reproduction thereof. The film was mounted between optical flats with suitable immersion oil in order to reduce phase differences due to uneven film thickness (Taylor \& Lipson, 1964). Since a rectangular aperture was used to define the tail sheath, the spots in the diffraction pattern arising from the aperture (at positions of the maxima of $(\sin x / x)^{2}$, namely, $x=1 \cdot 43 \pi, 2 \cdot 46 \pi, 3 \cdot 47 \pi, 4 \cdot 48 \pi, 5 \cdot 48 \pi, 6 \cdot 48 \pi, \ldots$, where $x=\pi a \sin \theta / \lambda, a$ being the slit width, $\theta$ being the scattering angle, and $\lambda$ being the wavelength of the light) could be used to calibrate dimensions on the diffraction pattern in terms of a scale on the original photograph.

An electron micrograph of a T4 phage with extended tail is shown in Plate $\mathrm{I}(\mathrm{a})$; the optical diffraction pattern of its tail sheath is shown in (b). Several features of this pattern are immediately interpretable. First, the very strong meridian spot (on the seventh layer-line) gives the annulus periodicity directly: this is 38.1 $A$. The optical diffraction pattern thus provides a direct measure of the periodicity in the structure and obviates the need for averaging measurements from the photograph. Second, since the $J_{0}$ Bessel function dominates the intensity distribution on the seventh layer-line, it is reasonable to suppose that the weaker satellite spots on either side of the strong meridian reflection represent maxima of $J_{0}^{2}(2 \pi R r)$, where $R$ is the radial co-ordinate in reciprocal space and $r$ the radius of the helix. Taking these to be the first maxima of $J_{0}^{2}$, we find $r=84 \AA$, in very good agreement with the outer diameter of the tail sheath as measured on electron micrographs. This also indicates that the stain has penetrated not far below the outer surface of the tail sheath. It should be noted that if stain has also penetrated to the inner diameter of the tail sheath, the corresponding $J_{0}^{2}$ reflection on the seventh layer-line would be expected at an $R$ value about 2.4 times that observed; this would probably be undetected in our diffraction pattern. Stain at this radius would, however, contribute significantly to altering the intensity distribution along other layer-lines at the $R$ 


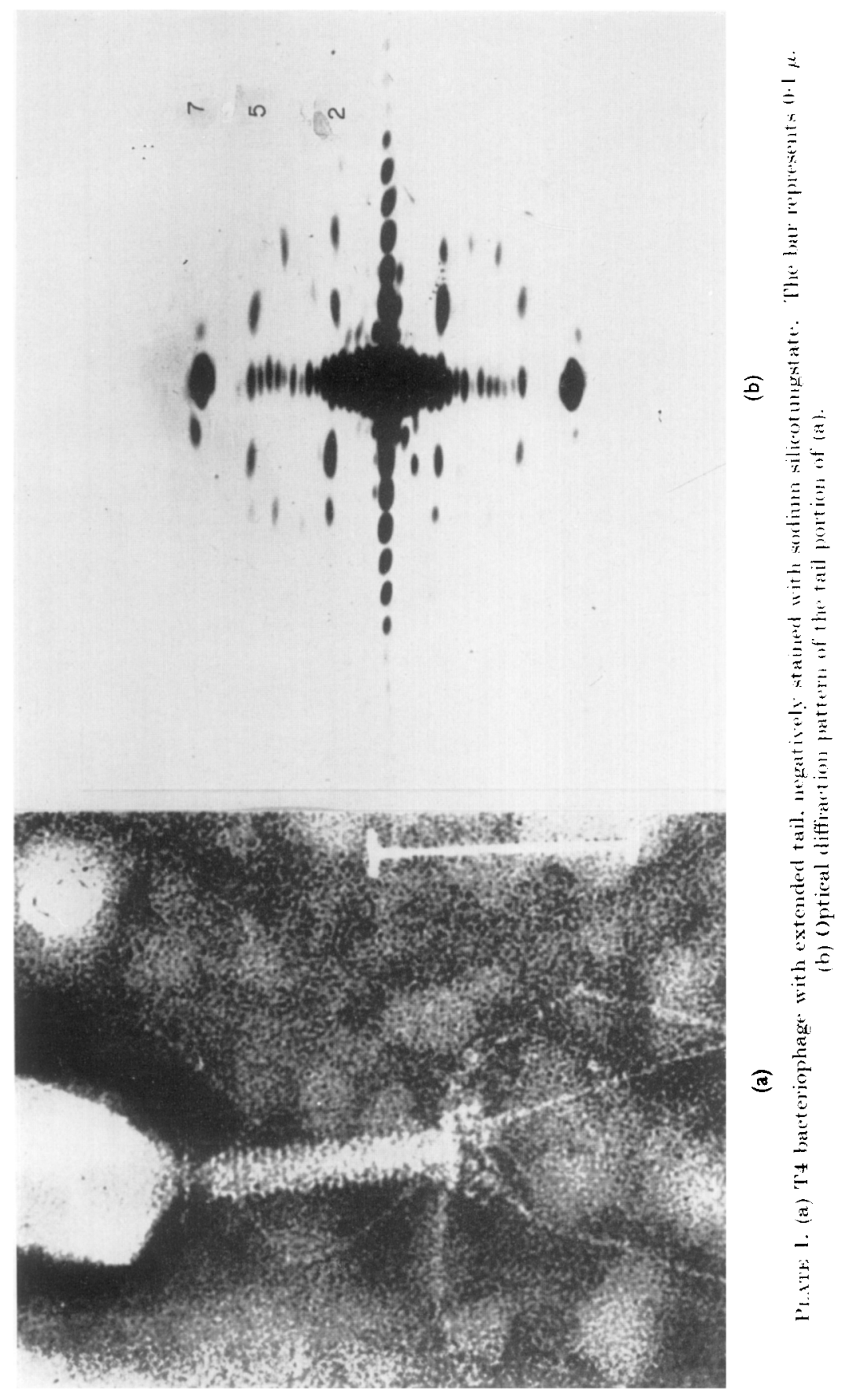




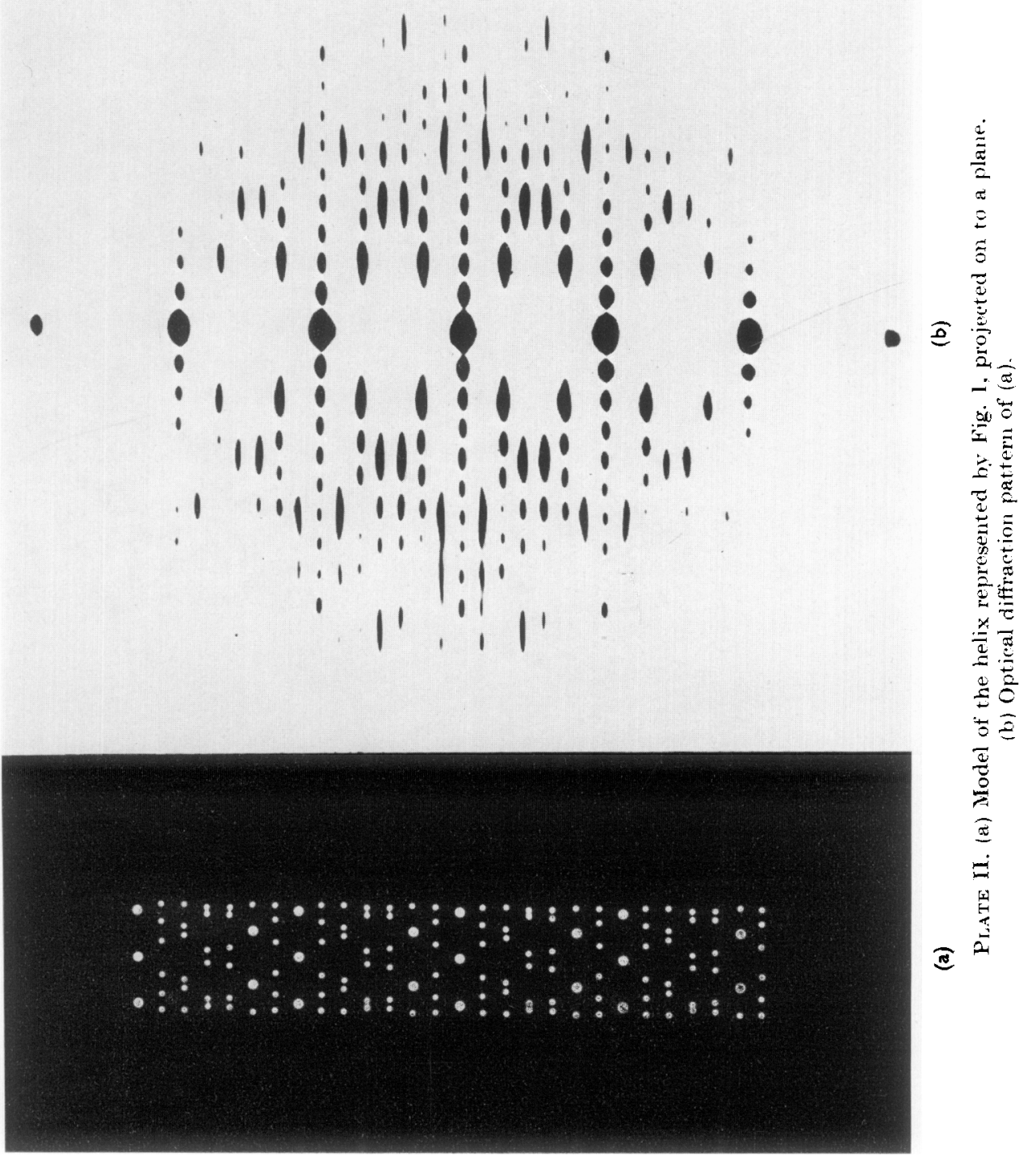




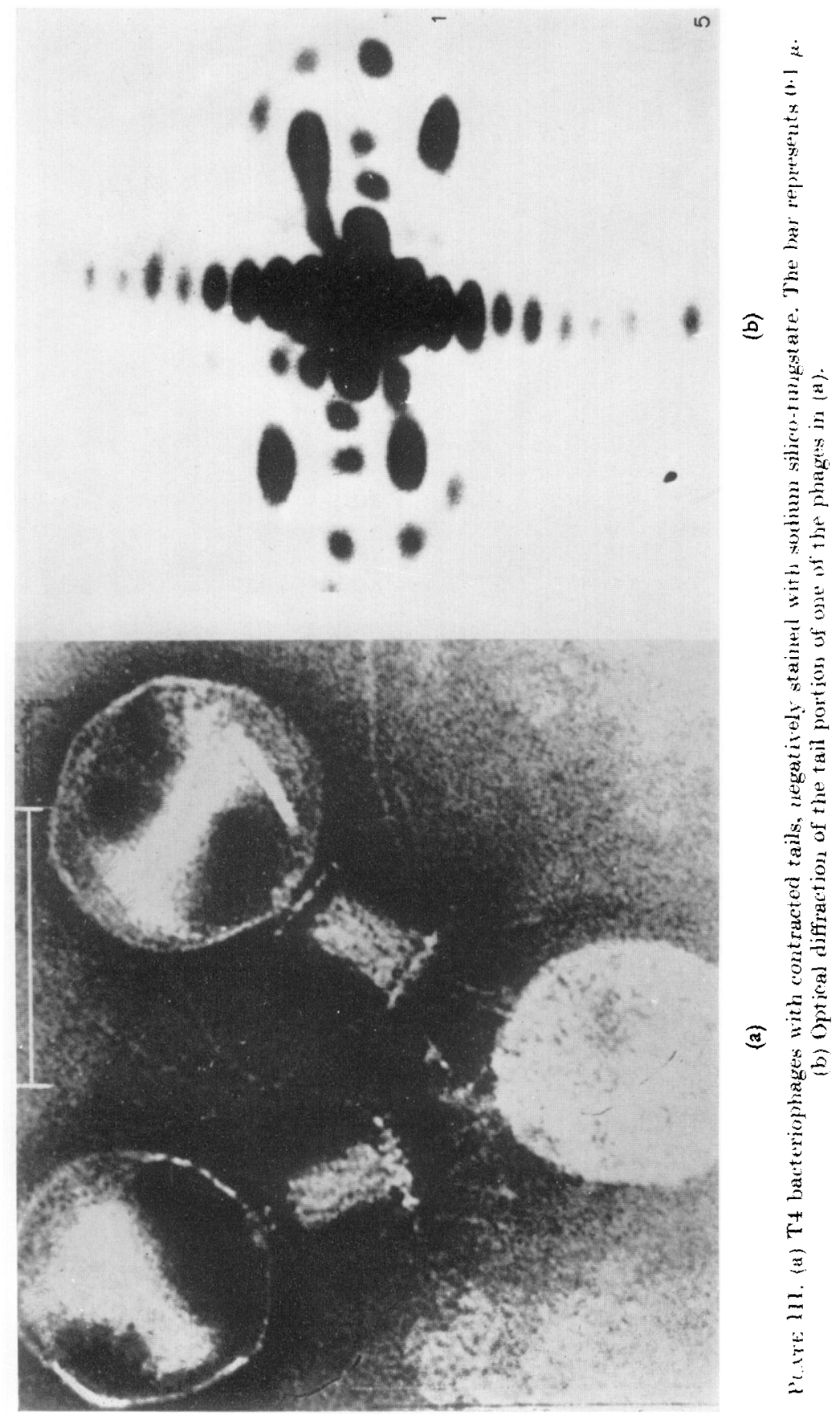




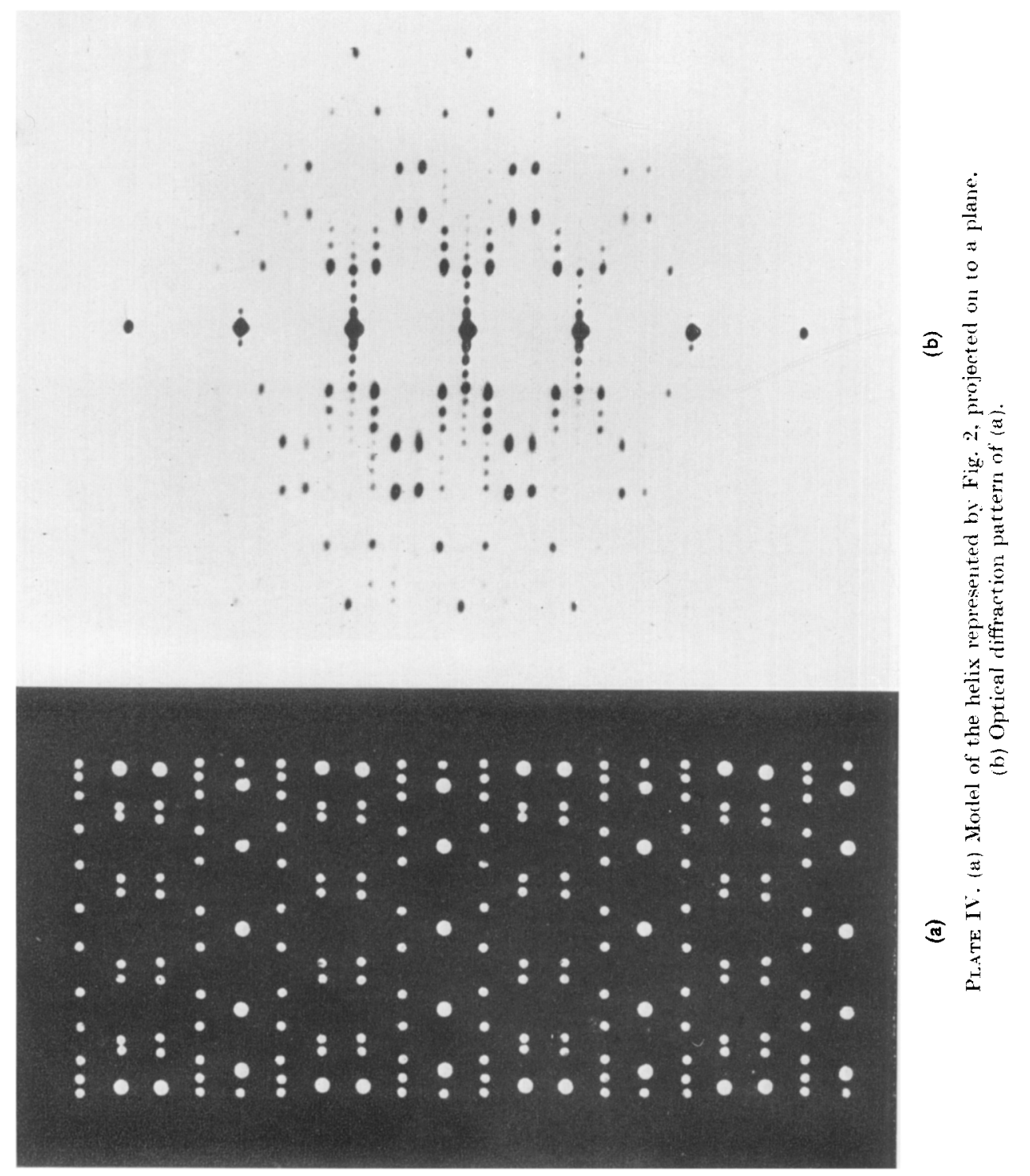


values where the outer surface stain is expected to give dominant reflections. Therefore, in the absence of knowledge about the distribution of stain in the tail sheath, the outer radius is most reliably determined from the inner subsidiary maximum of $J_{0}{ }^{2}$ on the seventh layer-line. Third, the meridional reflection is seen to occur on the seventh layer-line, indicating a helical repeat of $7 \times 38 \cdot 1=267 \AA$. (The measured layer-line spacings are multiples of $1 / 267 \AA^{-1}$ to within about $1 \%$ ). Fourth, the strong second layer-line reflections are seen not to be symmetrical in intensity about the meridian, a consequence of the uneven staining of the two sides of the sheath (Klug \& Berger, 1964). However, the weak and strong spots both have the same $R$ co-ordinate, showing that the extent of penetration of stain was not correlated with the amount of contrast developed by the stain. This is different from the case of tobacco mosaic virus (Klug \& Berger, 1964), and may indicate that the surface of the extended tail sheath does not have very deep depressions in it. Apparently little distortion of the structure has resulted from the staining procedure.

The helical symmetry of the subunit arrangement can be derived from the intensity distribution on the layer-lines. The strong contributions on layer-lines $l=\mathbf{2 , 3 , 4}$, and 5 , those on $l=2$ and 5 having the smaller $R$ values, are most consistent with a helix having close to seven units in two turns (the unit in this case being an annulus of six protein molecules). Alternatively, if we take the reciprocal lattice vectors to the strong spots on $l=2$ and $l=5$ as the two main lattice vectors, their relative orientation and size define the two-dimensional net of centers of subunits in a radial projection of the helix (Klug, Crick \& Wyckoff, 1958). This is shown in Fig. 1, and can be seen to correspond most closely to a helix of annuli with seven annuli in two turns. It is important to note that the net, when taken in conjunction with the known axial repeat and known radius, unambiguously specifies that each annulus contains six protein subunits. A model of such a helical structure is shown in projection in Plate II(a) and its optical diffraction pattern in (b). The similarity to Plate $\mathbf{I}(\mathbf{b})$ confirms the symmetry suggested above for the extended tail-sheath structure.

It is interesting to note that whereas the strong reflections on $l=2$ exhibit a marked intensity asymmetry, this is not true for the comparable spots on $l=5$. This would indicate that the helices defined by $A$ in Fig. 1 are separated by deeper grooves than those defined by B. As a consequence, the A grooves are not equally penetrated by stain on both sides of the sheath, resulting in an asymmetric intensity distribution on $l=2$; whereas stain penetrates the grooves between the $\mathrm{B}$ helices as easily on both sides of the tail sheath and gives a symmetric intensity distribution on $l=5$. This probably acoounts for the $\sim 60^{\circ}$ "stripcs" seen in electron micrographs (Kellenberger \& Boy de la Tour, 1964); the A helices in the model in Fig. 1 in fact make an angle of about $57^{\circ}$ with the base. It is important to note that the model in Fig. 1, when wrapped around into a cylinder, immediately accounts for the coarse helical structure often seen in electron micrographs (Bradley, 1963): these correspond to the A helices. It is not necessary to postulate subunits of different size or orientation (Bradley, 1963) in order to account for this feature in the electron micrograph. The subunit shape cannot be deduced from the above results, but it is worth observing that an ellipsoid of axes $40 \AA$ by $70 \AA$ (sce Fig. 1) would have a radial axis of $47 \AA$ (for a molecular weight of 54,000 and a density of 1.30), in good agreement with the measured thickness of the tail sheath (the subunit is probably more asymmetric than an ellipsoid, having a larger cross-section near the outer surface of the sheath than near the inner). 


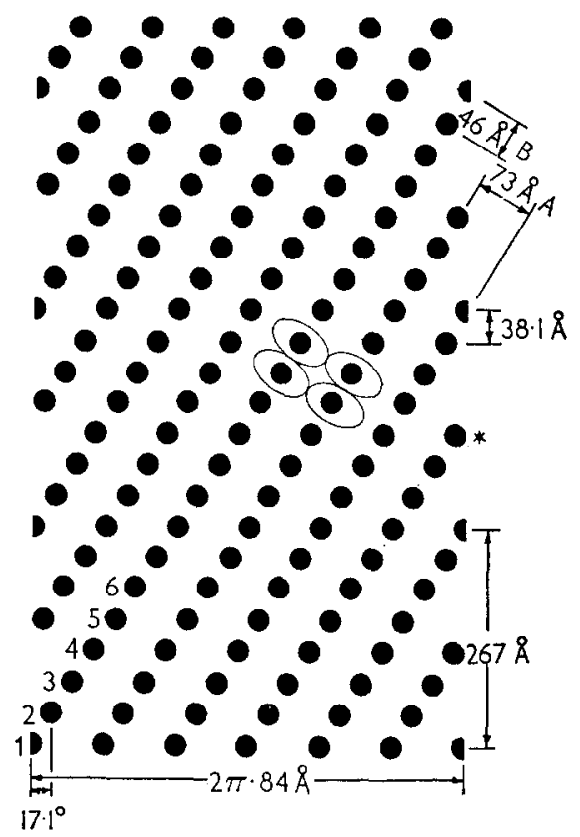

Fig. 1. Radial projection of the helix of 144 subunit centers of the extended tail sheath derived from the optical diffraction pattern of Plate $I(b)$.

An electron micrograph of T4 phages with contracted tails is shown in Plate III(a); the optical diffraction pattern of one such tail sheath is shown in (b). The pattern exhibits poorer order than was the case for the extended sheath, and this was true of all contracted sheaths examined. It is probable that the structure formed upon contraction is in general more poorly organized than that of the extended sheath. This is also suggested by the fact that the two pairs of strong layer-line spots nearest the origin no longer have the same $R$ co-ordinate, nor in fact identical axial spacings, indicating that negative staining has led to more relative distortion between the two sides of the contracted tail sheath than was the case for the extended sheath. The mean value of the unit periodicity determined from these layer-line reflections is about $160 \AA$. A weak meridional reflection nearest to the fifth layer-line suggests an axial repeat of about $32 \AA$. This diffraction pattern provides no information on the radius of the structure, so we will assume the value of about $130 \AA$ seen in electron micrographs.

Our inability to record unambiguously other reflections than those mentioned above prevents the direct determination of the lattice structure of the contracted tail sheath which was possible for the extended sheath. However, the above information in conjunction with knowledge of the extended sheath structure permits some reasonable inferences to be made. The observed contraction ratio of about $850 / 350=$ $2 \cdot 4$ shows that the $32 \AA$ periodicity in the diffraction pattern of the contracted sheath must have arisen from twice the $38.1 \AA$ repeat of the extended sheath pattern $(2 \times$ $38 \cdot 1 / 32=2 \cdot 4$ ). Thus the contracted sheath lattice cannot have arisen from the extended sheath merely by an intact tilting of the helices marked A in Fig. 1, (otherwise the $38 \cdot 1 \AA$ repeat would collapse to $16 \AA$ ). The contracted sheath lattice must 
result from a merging of pairs of annuli in the extended sheath. It is interesting to note that if this is done, then the 11 th annulus (marked $*$ in Fig. 1 ) is only $9^{\circ}$ away from coinciding with the first annulus, and a helix with five units (annuli) per repeat can result naturally with a minimal amount of rotation of protein subunits about the tail sheath axis. The undistorted lattice involving such alternate annuli of Fig. 1 (i.e., 1,3,5. . . ) is shown in Fig. 2 by filled circles. The strong first layer-line reflections in Plate III(b) arise from the B helices in Fig. 2, and their equal intensities indicate that the grooves between these helices are shallow (and thus equally penetrated by stain on both sides of the tail sheath).

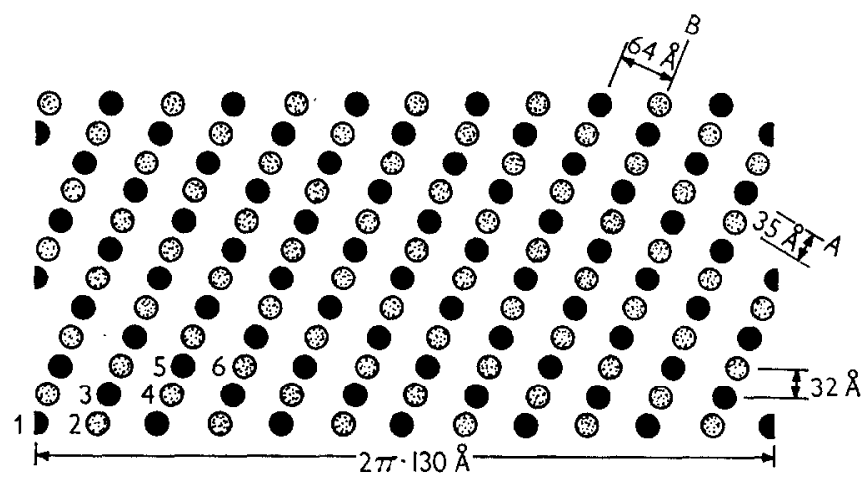

Frg. 2. Radial projection of the helix of 144 subunit centers of the contracted tail sheath. Units numbered $1,2,3,4,5$ and 6 correspond to similarly numbered units in Fig. 1.

The position of the remaining subunits is diffeult to determine from the diffraction pattern alone, but we think that they are most likely to be uniformly interspersed between the subunits of the above annuli, as indicated by the stippled circles of Fig. 2. The contracted sheath thus consists of annuli containing 12 subunits, in contrast with six/annulus for the extended sheath. Such a structure with 12 subunits/ annulus can also be inferred from axial electron microscope views of individual contracted sheath annuli (Anderson, 1963). A model of such a structure is shown in projection in Plate IV(a) and its diffraction pattern in (b). The observed pattern (Plate III(b)) is at least consistent with this structure in that no significant diffraction is seen near the meridian above the first layer-line. The absence of observed reflections at higher $R$ values for layer-lines above the first may be due to the poor order which, as we noted earlier, seems to characterize the contracted sheath structure. This may also account for the weakness (compared to the model) of the observed meridional reflection. Aside from such diffraction considerations, the structure of Fig. 2 seems compelling also because it retains the equivalence of subunits which we know exists in the structure of the extended sheath. It is interesting to note that the $A$ helices in Fig. 2 make an angle of about $30^{\circ}$ with the base, thus accounting for the "stripes" often seen in electron micrographs at roughly this angle (Kellenberger \& Boy de la Tour, 1964).

\section{Conclusions}

The above structures of the extended and contracted sheaths suggest that the mechanism of contraction of the tail sheath involves a conformational change in the 
protein subunit. This is indicated by the dimensional changes in the subunit which are required to fit the above lattices; namely, the increase in the radial dimension (assuming the inner radius of the contracted sheath to be relatively unchanged) and the decrease in the other two dimensions which is suggested by the lattice of Fig. 2. Such a conformational change could be triggered by the hydrolysis of ATP to form ADP which is known to accompany contraction, since there are as many ATP molecules as protein subunits (Wahl \& Kozloff, 1962). Indeed, optical rotatory dispersion measurements indicate that a change in conformation of the polypeptide chain accompanies the contractile process (Poglazov \& Tikhonenko, 1963). The conformational change may well alter the disposition of interaction sites on a protein subunit, thus changing the geometry of its interaction with neighboring subunits. Thus, while the interaction may be between adjacent units along the A helices of the extended sheath, as perhaps indicated by the shallow grooves between the B helices in Fig. 1, this might shift to a lateral interaction between the subunits of each annulus of the contracted sheath, which would be consistent with the shallow grooves indicated between the B helices in Fig. 2. It is interesting to speculate that, in order that the proposed pairing of annuli suggested above shall not isolate single annuli, it may be necessary that the pairing start from one end of the tail sheath and progress uniformly to the other end. This may be an example of what could be called a "domino reaction," namely, a sequential change which is propagated along a onedimensional path as a result of the reaction in a given unit of the chain initiating a similar reaction in the succeeding unit.

This research was supported by grants to the authors from the U.S. Public Health Service, AM-02830 (S. K.) and CA-06927 (T.F.A.), and from the National Science Foundation, GB.982 (T. F. A.).

\section{REFERENCES}

Anderson, T. F. (1960). Proc. Eur. Reg. Conf. on Electron Microscopy, vol. 2, p. 1008.

Anderson, T. F. (1963). In Viruses, Nucleic Acids and Cancer, p. 122. Baltimore: Williams \& Wilkins.

Anderson, T. F. \& Stephens, R. (1964). Virology, 23, 113.

Bradley, D. E. (1963). J. Gen. Microbiol. 31, 435.

Brenner, S. \& Horne, R. W. (1959). Biochim. biophys. Acta, 34, 103.

Brenner, S., Streisinger, G., Horne, R. W., Champe, S. P., Barnett, L., Benzer, S. \& Rees, M. W. (1959). J. Mol. Biol. 1, 281.

Daems, W Th., Van de Pol, J. J. \& Cohen, J. A. (1961). J. Mol. Biol. 3, 225.

Fernández-Morán, H. (1962). In The Interpretation of Ultrastructure, ed. by R. J. C. Harris, vol. 1, p. 411. New York: Academic Press.

Kellenberger, E. \& Boy de la Tour, E. (1964). J. Ultrastructure Res. 11, 545.

Klug, A. \& Berger, J. E. (1964). J. Mol. Biol. 10, 565.

Klug, A., Crick, F. H. C. \& Wyckoff, H. W. (1958). Acta Cryst. 11, 199.

Poglezov, B. F. \& Tikhonenko, A. S. (1963). Biochimiya, 28, 888.

Sarkar, N., Sarkar, S. \& Kozloff, L. M. (1964). Biochemistry, 3, 511.

Taylor, C. A. \& Lipson, H. (1964). Optical Transforms. London: G. Bell and Sons, Ltd.

Wahl, R. \& Kozloff, L. M. (1962). J. Biol. Chem. 237, 1953. 